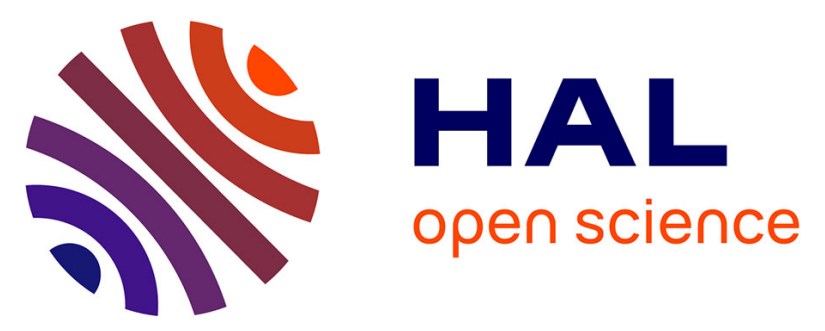

\title{
Fiber optic refractive index sensors through spectral detection of Rayleigh backscattering in a chemically etched $\mathrm{MgO}$-based nanoparticle-doped fiber
}

Marzhan Sypabekova, Sanzhar Korganbayev, Wilfried Blanc, Takhmina Ayupova, Aliya Bekmurzayeva, Madina Shaimerdenova, Kanat Dukenbayev, Carlo Molardi, Daniele Tosi

\section{To cite this version:}

Marzhan Sypabekova, Sanzhar Korganbayev, Wilfried Blanc, Takhmina Ayupova, Aliya Bekmurzayeva, et al.. Fiber optic refractive index sensors through spectral detection of Rayleigh backscattering in a chemically etched MgO-based nanoparticle-doped fiber. Optics Letters, 2018, 43 (24), pp.5945. hal-01985843

\section{HAL Id: hal-01985843 https://hal.science/hal-01985843}

Submitted on 18 Jan 2019

HAL is a multi-disciplinary open access archive for the deposit and dissemination of scientific research documents, whether they are published or not. The documents may come from teaching and research institutions in France or abroad, or from public or private research centers.
L'archive ouverte pluridisciplinaire HAL, est destinée au dépôt et à la diffusion de documents scientifiques de niveau recherche, publiés ou non, émanant des établissements d'enseignement et de recherche français ou étrangers, des laboratoires publics ou privés. 


\title{
Fiber optic refractive index sensors through spectral detection of Rayleigh backscattering in a chemically etched MgO-based nanoparticle-doped fiber
}

\author{
Marzhan Sypabekova, ${ }^{1,2}$ Sanzhar Korganbayev, ${ }^{1}$ Wilfried Blanc, ${ }^{3}$ Takhmina Ayupova, ${ }^{1}$ \\ Aliya Bekmurzayeva, ${ }^{1,2}$ Madina Shaimerdenova, ${ }^{1}$ Kanat Dukenbayev, ${ }^{2}$ Carlo Molardi, ${ }^{2}$ \\ AND DANIELE TosI ${ }^{1,2, *}$ \\ ${ }^{1}$ National Laboratory Astana, Laboratory of Biosensors and Bioinstruments, 010000 Astana, Kazakhstan \\ ${ }^{2}$ Nazarbayev University, Department of Electrical and Computer Engineering, 010000 Astana, Kazakhstan \\ ${ }^{3}$ Université Côte d'Azur, INPHYNI-CNRS UMR 7010, Parc Valrose, 06108 Nice, France \\ *Corresponding author: daniele.tosi@nu.edu.kz
}

Received 18 October 2018; revised 2 November 2018; accepted 9 November 2018; posted 9 November 2018 (Doc. ID 348705); published 4 December 2018

We demonstrate and experimentally validate a fiber optic refractive index (RI) sensor obtained by simply etching a high-scattering $\mathrm{MgO}$-based nanoparticle-doped single-mode fiber in hydrofluoric acid (HF). The fiber has $32.3 \mathrm{~dB}$ stronger Rayleigh scattering than a standard fiber, allowing a detection of scattering spectral signatures with an optical backscatter reflectometer, even when the core is exposed to the outer RI. The obtained sensitivity is $1.53 \mathrm{~nm} / \mathrm{RIU}$ (RI units), measured by correlating the scattering spectra. We prove the possibility of implementing a distributed RI detection (seven locations spaced by $1 \mathrm{~mm}$ ). The fabrication method for this RI sensor is simplified, since it simply requires etching in an HF bath, without the need of inscribing reflective elements or fabricating microstructures in the fiber.

https://doi.org/10.1364/OL.43.005945

Optical fiber refractive index (RI) sensors allow the measurement of the RI external to the fiber probe, and they exhibit high sensitivity and a compact form factor [1]; RI sensors are also the building blocks of fiber optic biosensors, which allow the measurement of biological analytes through a suitable functionalization [2-6]. The majority of fiber optic biosensors encodes the sensing principle in the variations of the optical spectrum of the sensing element [1-6], fabricated within the fiber or enclosed around it. This method of detection is reliable, as it allows a detection independent of the power level fluctuations.

Since the light propagates within the core of a standard fiber to facilitate the interaction with the RI surrounding the fiber, several methods have been proposed, which allow the confinement factor reduction and the evanescent wave enhancement in the sensing region [2]. Several modern RI sensors make use fiber Bragg grating (FBG)-based devices [1] such as tilted FBGs (TFBGs) [3,4], etched FBGs [7,8], and long-period gratings (LPGs) [9]. The main alternative to devices based on gratings is composed of optical microstructures such as fiber tapers [5,6] or plasmonic devices [10], sometimes facilitated by tilted gratings $[3,4]$.

These architectures allow high sensitivity and are suitable for biological functionalization [3-6]. However, they have the drawback of requiring a complex fabrication. Grating-based RI sensors require the inscription of an FBG in the fiber, using a phase mask or femtosecond laser inscription method [1]. Plasmonic devices imply the generation of surface plasmon resonance (SPR) waves in the fiber, which requires fabrication of nano-layers $[3,4,10]$. Fiber tapers, as well as LPGs, can be fabricated using a fiber splicer [5,6]. In addition, several sensors based on SPR, LPGs, and TFBGs work in transmission; to back-reflect the transmission spectrum from the sensing region, the fabrication of additional gold-tip mirrors is required [3].

The fabrication of RI sensors in optical fiber is a complex operation, which requires photo-inscription and/or microstructures and, therefore, limits the possibility of volume manufacturing. However, chemical etching such as wet-etching in a hydrofluoric acid (HF) bath, is a simple operation, frequently used in mass manufacturing of optical [7] and electronic devices $[11,12]$.

In this Letter, we demonstrate the fabrication of an RI sensor by simply etching a special nanoparticle-doped single-mode fiber (SMF) and analyzing the reflection spectrum of the Rayleigh backscattering with an optical backscatter reflectometer (OBR) [13,14]. In this framework, wet-etching is the only fabrication step involved, as the reflective device is the scattering pattern itself. When the fiber is etched and exposed to the outer RI, we observe a shift of the random scattering spectra, 

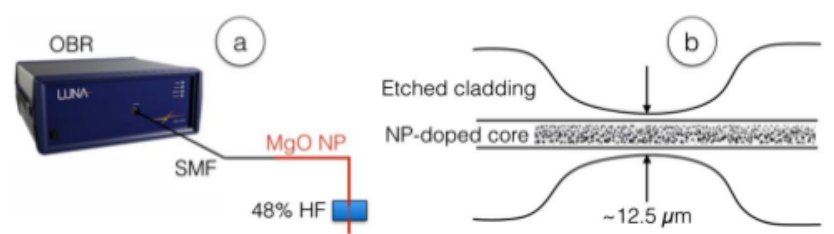

Fig. 1. Schematic of the sensor interrogation and etching.

thus encoding the demodulation in the shift of optical reflection spectra as in most aforementioned sensors.

The experimental setup of the system is described in Fig. 1. The analyzer is an OBR system (Luna, Inc., OBR 4600) operating with a gauge length of $1 \mathrm{~mm}$, a detection range of $26 \mathrm{~mm}$, and a wavelength window between 1545 and $1588 \mathrm{~nm}$. The operation is a distributed sensing mode with a speed of $3 \mathrm{~Hz}$. The output of the OBR is connected to the MgO-based nanoparticle-doped sensing fiber $(40 \mathrm{~cm})$ through an SMF spool; SMF and nanoparticle-doped fibers have been spliced using a standard splicer (Fujikura 12-S, SMF-SMF splicing mode). The nanoparticle-doped fiber has been etched about $5 \mathrm{~mm}$ away from the tip, by immersing it for $220 \mathrm{~min}$ in a solution of $48 \%$ HF. Etching has been performed putting the fiber in an $\mathrm{HF}$ bath having $\sim 1 \mathrm{~cm}$ of diameter, in a chemical fume hood (Waldner Secuflow airflow controller, ceiling-bench mounted), maintaining the ambient temperature. The OBR has been used to measure the loss pattern during the whole etching process. The same quality HF solution has been used throughout the whole etching process in a sealed container. Once the OBR started to detect the loss pattern, the fiber has been placed in a less concentrated $5 \%$ HF solution to slow down the etching and, hence, allowing controlled completion of the process.

The fiber, used as an RI sensor, was originally drawn to be an efficient optical amplifier operating in a C-band (1530 $1565 \mathrm{~nm}$ ) showing, in the core area, a doping of erbium and $\mathrm{MgO}-$ based nanoparticles. For this reason, the fiber presents the typical size of a telecom fiber, with a core diameter of $10 \mu \mathrm{m}$ and a cladding diameter of $125 \mu \mathrm{m}$. Nanoparticles present a random size between 20 and $100 \mathrm{~nm}$, and their distribution follows a random pattern. The size and distribution of nanoparticles are given by the technological procedure used to fabricate this kind of fiber. The preform, fabricated with the modified chemical vapor deposition (MCVD) process, presents a porous substrate of silica doped with a solution of $\mathrm{MgCl}_{2}$. During the heating process, i.e., sintering of the porous layer and collapsing of the tube, the spontaneous phase separation between silica and the alkaline ion occurs, creating two phases: one rich with silica and one made of $\mathrm{MgO}$-rich spherical particles $[15,16$. The content of $\mathrm{MgO}$ in each particle varies according to the random process of phase separation. Each particle, therefore, is a compound of silica substrate, $\mathrm{MgO}$, and other components such as germanium and erbium. This in situ process does not require a further heat treatment to the final fiber in order to form nanoparticles. The characteristics of the nanoparticles are dependent on the $\mathrm{Mg}$ concentration. By controlling the preform doping, parameters such as particle density and particle average size can be varied [16]. This makes it possible to tailor the Rayleigh scattering of the fiber, thus changing the spatial operation window of the sensor.

In Fig. 2, the scattering pattern of the nanoparticle-doped fiber is shown, reporting the backscattered power as a function

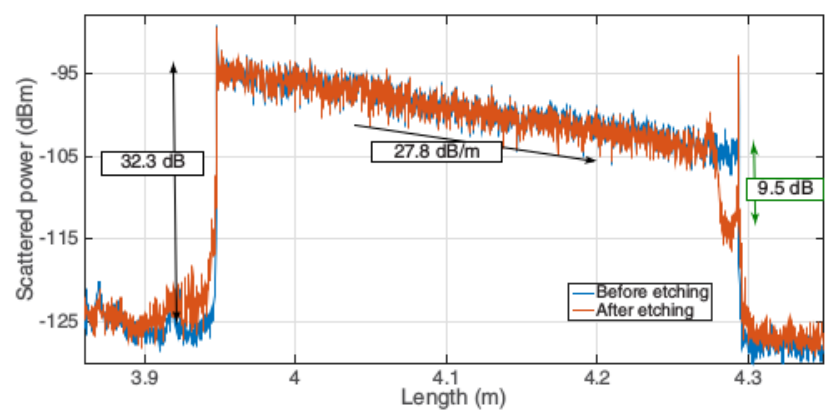

Fig. 2. Scattering trace of the $\mathrm{MgO}$-based nanoparticle-doped fiber before (blue line) and after (red line) etching.

of the length. The lead-in SMF has a length of $3.95 \mathrm{~m}$, and the average backscattered power is $126.4 \mathrm{dBm}$, which is just higher than the limit of detection of the OBR, about $130 \mathrm{dBm}$ [13]. Into the $\mathrm{MgO}$-doped fiber, we observe a steep increment of the backscattered power, which is $-94.1 \mathrm{~dB}$, just after the SMF/ $\mathrm{MgO}$ splice. This corresponds to a "gain" of $32.3 \mathrm{~dB}$, which is not an amplification factor, but it defines how much higher the scattering trace appears on the OBR than a standard SMF. The fiber has a higher attenuation of $27.8 \mathrm{~dB} / \mathrm{m}$ (which corresponds to twice the fiber attenuation, considering that losses affect both forward and back-reflected waves). Prior to fiber etching, we observe a sawtooth-shaped waveform; when the fiber is etched in correspondence with the tip, we observe a decrease of the reflected power due to the effect of the reduced confinement factor [7]. To some extent, this is the same working principle of thinned FBGs. The losses induced on the etched regions are not perfectly constant, since each fiber section has a different diameter; however, we can observe that the difference in scattering traces before and after etching is $9.5 \mathrm{~dB}$ in the point of maximum sensitivity.

We remark that, for this RI sensor, based on evanescent waves, it is necessary to use a fiber having high scattering: an SMF fiber would not be able to sustain a loss of $9.5 \mathrm{~dB}$ (or higher, for superior RI sensitivity) without falling below the OBR limit of the detection threshold. Instead, by using an $\mathrm{MgO}$-based nanoparticle-doped fiber the OBR sweptwavelength interferometers operate with a backscattering power of $-115 \mathrm{dBm}$, over $10 \mathrm{~dB}$ higher than an SMF, despite the combined effect of fiber attenuation and evanescent wave losses.

The fiber profile is shown in Fig. 3. The first chart shows the scanning electron microscope (SEM) view of the fiber core cross section prior to etching, which shows the effect of $\mathrm{MgO}-$ based nanoparticles into the inner portion of the core. The nanoparticles are found in a diameter of about $9.5 \mu \mathrm{m}$ within the core, and they do not reach the cladding. The second surface nanoscopic characterization has been performed on the fiber after its etching process, using high-resolution atomic force microscopy (AFM, SmartSPM 1000 AIST-NT). Surface scanning of the etched fiber was made using a non-contact mode, operating in air. We used high-accuracy non-contact etalon cantilever with a tip radius less than $10 \mathrm{~nm}$ and force constant of $17 \mathrm{~N} / \mathrm{m}$ (TipsNano). The profile cross-sectional image was built by plotting five curves measured throughout whole scanned area that allows estimating the etched diameter to about $12.5 \mu \mathrm{m}$, which implies that the high scattering content 


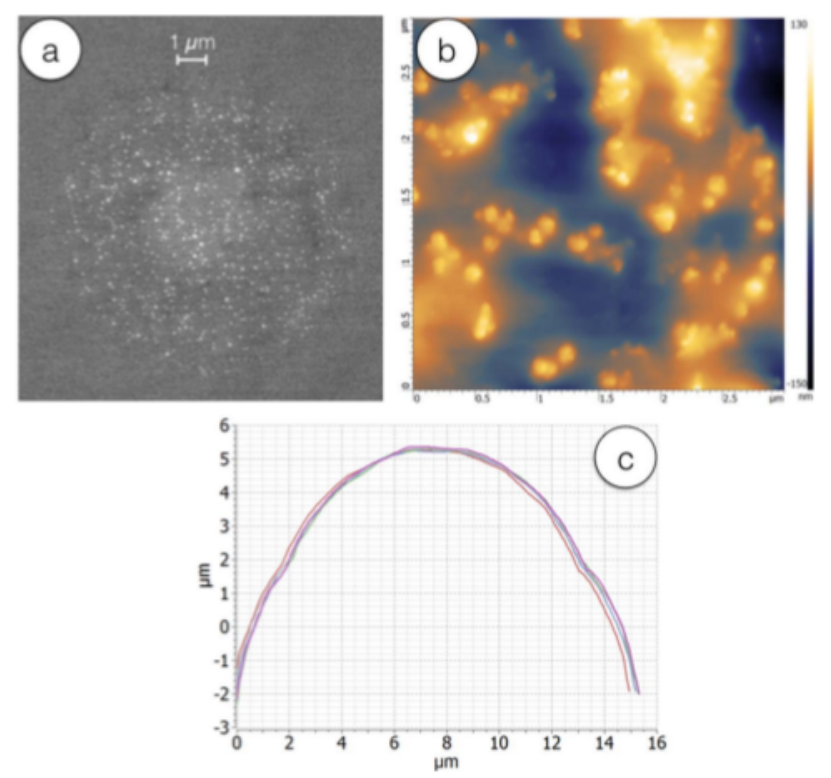

Fig. 3. (a) SEM view of the fiber core cross section prior to etching. (b) AFM topographical view of the external side of the fiber after etching; the image size is equal to $3 \times 3 \mu \mathrm{m}$, and the height ranges from -150 to $130 \mathrm{~nm}$. (c) AFM profilometry, measuring the fiber thickness in five different sections.

is located only within the core and does not affect light propagation near the cladding interface, where the change of effective RI is experienced. As a confirmation, Figure 3(b) shows the top image of the fiber outer portion measured with AFM: we can observe surface defects having similar profiles to $[7,8]$, with defects that have size much larger than the MgO-based nanoparticles, two-10 times larger comparing Fig. 3(a) with Fig. 3(b); this confirms that the etching performance is similar to thinned fibers also in terms of superficial defects, and the MgO-doping does not affect the wet-etching process.

The RI sensitivity has been evaluated as in Refs. [1,7], by immersing the etched fiber into a sucrose/water solution, mixed in a stirrer (Waldner Secuflow airflow controller, ceiling-bench mounted). All solutions are held at room temperature in a chemical fume hood with temperature stabilization $\pm 0.1^{\circ}$ (Waldner Secuflow airflow controller, ceiling-bench mounted). In the etched section, we observe a change of effective RI which is a function of the external RI surrounding the fiber. This, in turn, has two effects: (1) the scattered power loss within the etched region depends on the external RI; (2) we observe a wavelength shift of the Rayleigh signature, i.e., the spectrum of the local Rayleigh scattering pattern, similar to the one observed in the OBR by a fiber exposed to temperature or strain change $[13,14]$.

In terms of sensing capability, the first effect is weaker, since it implies demodulating the intensity of the backscatter signal; however, this is less tolerant to the fiber losses in the long-term and, in general, the backscatter power is a noisy signal. It is preferable to encode the sensing mechanism in the optical spectrum, whereas wavelength shifts are more tolerant to power fluctuations [2] and, for this reason, we can analyze the spectral shift of the fiber signature.

In Fig. 4(a), an inset of the fiber signatures is shown, where the fiber is in the reference condition ( $0 \%$ sucrose) and with an
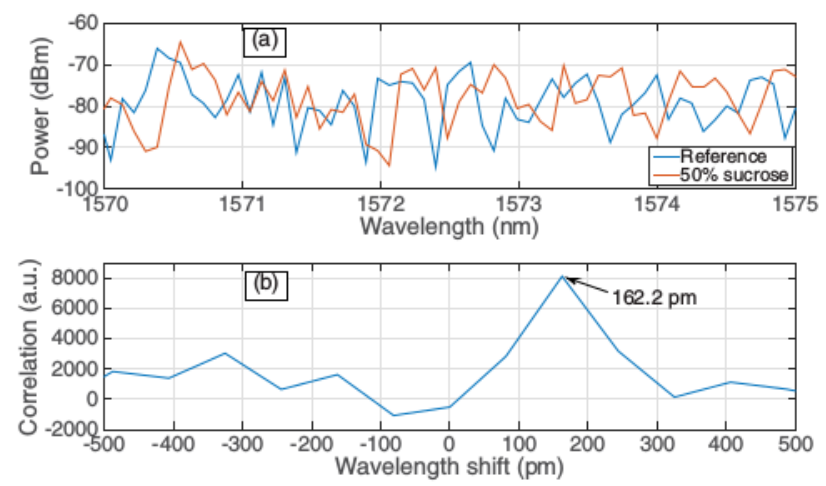

Fig. 4. Analysis of the fiber signature when the etched $\mathrm{MgO}$-doped fiber is exposed to an RI change. (a) Spectrum of the wavelength shift in the reference condition (fiber in water) and in the measurement condition ( $50 \%$ sucrose). (b) Mutual correlation between the measured and reference signatures, evaluating the wavelength shift.

RI change ( $50 \%$ sucrose). The signature is calculated at the location $4.279 \mathrm{~m}$. We observe that the signature has the classical random pattern, due to the fiber defects, and it is similar to $[13,17]$; the nanoparticles in the core enhance the scattered power, but since their distribution and size are random, they maintain a random spectral signature. When the surrounding RI changes, we observe a shift of the backscattered signal, which is used by the OBR to detect the wavelength shift [17]. The signature has been acquired on the 1545-1588 nm range with $8 \mathrm{pm}$ grid; we report a portion that better highlights the behavior. Figure 4(b) shows the estimation of the wavelength shift, which has been performed by using the classical OBR algorithm that calculates the peak of the mutual correlation between the reference signature and the measured signature (subtracting the mean value from each signature). The result is shown in the chart and has a clearly detectable peak in correspondence of a wavelength shift of $162.2 \mathrm{pm}$, which corresponds to the shift that can be deducted from the signatures.

The mutual correlation is a robust algorithm, because it is independent of the signal level, since the output is the wavelength at which the correlation is at the maximum; thus, effects such as power fluctuations or RI-induced losses do not affect the estimate.

By immersing the fiber into multiple water/sucrose concentrations $(6.25 \%, 12.5 \%, 25 \%$, and $50 \%)$ at constant room temperature, we can evaluate the RI sensitivity by estimating the wavelength shift of the fiber signature for different RI values. The result is shown in Fig. 5, where an almost linear sensitivity of $1.53 \mathrm{~nm} / \mathrm{RIU}$ is observed. This value is similar to Refs. $[7,18]$ and inferior to Refs. $[9,10]$; however, it is possible to raise the sensitivity by $1-2$ orders of magnitude by further reducing the fiber diameter [7].

$\mathrm{OBR}$ is a technique for distributed sensing, as it allows estimating the signature at each fiber location [14]. We performed an experiment immersing the whole etched fiber in different values of the RI and estimating the RI sensitivity on each section. The gauge length, i.e., two adjacent locations at which the signatures are estimated, has been set to $1 \mathrm{~mm}$ which corresponds to a dense sensing (10 detection points along the etched portion of the nanoparticle-doped fiber). The result is shown in Fig. 6 for the etched section 


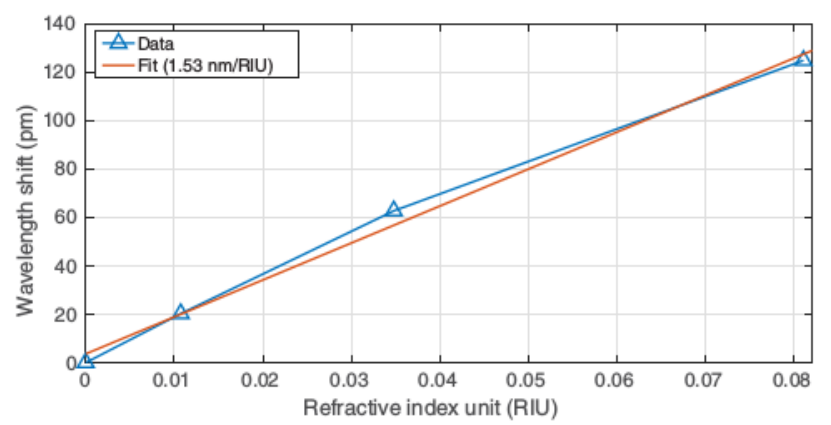

Fig. 5. Wavelength shift of the Rayleigh scattering signature as a function of the RI change.

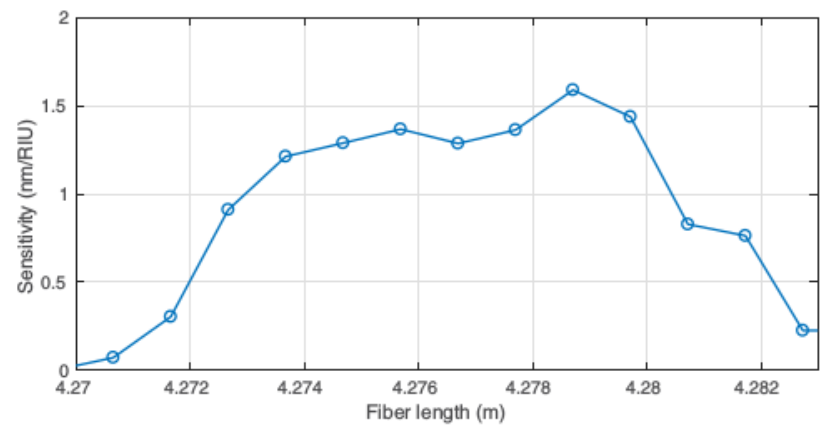

Fig. 6. Refractive index sensitivity estimated at each location of the etched fiber.

$(4.270-4.282 \mathrm{~m})$. The maximum sensitivity approaches $1.6 \mathrm{~nm} / \mathrm{RIU}$ in correspondence of the thinnest diameter. Each section has a slightly different fiber diameter, and the sensitivity changes in space; however, we observe an inner plateau with sensitivity higher than $1.2 \mathrm{~nm} / \mathrm{RIU}$ in correspondence of $4.273-4.279 \mathrm{~m}$ (seven locations). Then the sensitivity decays outside the inner region, following the etching profile.

Overall, this proof of concept demonstrates the possibility of combining OBR with an etched fiber for distributed RI detection. For possible applications, this method inherently has a low-cost fiber sensor, which is considered as a disposable in biomedical applications. Fiber etching is the only fabrication involved, and there is no need for fabricating a microstructure or a grating reflector. The fact that the $\mathrm{MgO}$-based nanoparticledoped fiber can be easily spliced to an SMF fiber using the SMF/SMF splicing mode allows limiting the sensing length to the only sensing device. With the parameters estimated as in Fig. 2, and assuming a $\sim 20 \mathrm{~dB}$ loss due to evanescent waves in a high-sensitive device [7], the backscattered power is higher than the OBR detection threshold for a sensor length equal to $1.3 \mathrm{~m}$, which virtually enables a true distributed RI sensing over tens of centimeters at millimeter-scale resolution.

In conclusion, we reported the realization of a refractometer based on etching an MgO-based nanoparticle-doped SMF, having high scattering content (32.3 dB higher scattering). The sensor does not require the inscription of any grating or reflective device and does not require fiber micro-fabrication, as wet-etching is the only process involved. Furthermore, the nanoparticle-doped fiber fabrication is based on an established
MCVD technique, which does not require additional fabrication processes. Therefore, fabrication is simplified. This represents a benefit in terms of cost associated to the technological process. We make use of the Rayleigh backscattering that is observed in the fiber as a reflective element, whose effective RI changes with the surrounding RI. Demodulation has been implemented by evaluating the mutual correlation of Rayleigh backscattering signatures, as in classical OBR systems. Thanks to the high scattering of the fiber, it is possible to perform a correct detection of the scattering signatures, despite the extra attenuation due to evanescent waves. A sensitivity of $1.53 \mathrm{~nm} /$ RIU has been achieved, with the possibility of performing a distributed RI detection throughout the whole length of fiber exposed by etching. We remark that the proposed method is also prone for temperature compensation, since the unetched portion of the fiber maintains its original thermal sensitivity, but has no RI dependence.

Future work will be addressed to use the methodology to create a longer distributed refractometer, to functionalize the fiber for biosensing, and to engineer the scattering profile of the fiber in order to raise the scattering increment and lower attenuation figure.

Funding. ORAU Programme at Nazarbayev University (FOSTHER, LIFESTART); project Nice-DREAM, Agence Nationale de la Recherche (ANR) (ANR-14-CE07-0016-03).

\section{REFERENCES}

1. W. Liang, Y. Huang, Y. Xu, R. K. Lee, and A. Yariv, Appl. Phys. Lett. 86, 151122 (2005).

2. A. Leung, P. M. Shankar, and R. Muthasaran, Sens. Actuators, B 125, 688 (2007).

3. C. Ribaut, M. Loyez, J. C. Larrieu, S. Chevineau, P. Lambert, M. Remmelink, R. Wattiez, and C. Caucheteur, Biosens. Bioelectron. 92, 449 (2017).

4. C. Caucheteur, Y. Shevchenko, L. Y. Shao, M. Wuilpart, and J. Albert, Opt. Express 19, 1656 (2011).

5. D. Sun, T. Guo, Y. Ran, Y. Huang, and B. O. Guan, Biosens. Bioelectron. 61, 541 (2014).

6. S. Gao, L. P. Sun, J. Li, L. Jin, Y. Ran, Y. Huang, and B. O. Guan, Opt. Express 25, 13305 (2017).

7. A. ladicicco, A. Cusano, S. Campopiano, A. Cutolo, and M. Giordano, IEEE Sens. J. 5, 1288 (2005).

8. L. Coelho, J. M. de Almeida, J. L. Santos, and D. Viegas, Appl. Opt. 54, 10342 (2015).

9. F. Esposito, L. Sansone, C. Taddei, S. Campopiano, M. Giordano, and A. ladicicco, Sens. Actuators, B 274, 517 (2018).

10. S. Cao, Y. Shao, Y. Wang, T. Wu, L. Zhang, Y. Huang, F. Zhang, C. Liao, J. He, and Y. Wang, Opt. Express 26, 3988 (2018).

11. G. A. Spierings, J. Mater. Sci. 28, 6261 (1993).

12. D. J. Monk, D. S. Soane, and R. T. Howe, Thin Solid Films 232, 1 (1993).

13. B. J. Soller, D. K. Gifford, M. S. Wolfe, and M. E. Froggatt, Opt. Express 13, 666 (2005).

14. M. Froggatt and J. Moore, Appl. Opt. 37, 1735 (1998).

15. J. B. M. Chesney, P. B. O'Connor, and H. M. Presby, Proc. IEEE 62, 1280 (1974).

16. W. Blanc, V. Mauroy, L. Nguyen, B. N. Shivakiran Bhaktha, P. Sebbah, B. P. Pal, and B. Dussardier, J. Am. Ceram. Soc. 94, 2315 (2011).

17. F. Parent, S. Loranger, K. K. Mandal, V. L. lezzi, J. Lapointe, J. S. Boisvert, M. D. Baiad, S. Kadoury, and R. Kashyap, Biomed. Opt. Express 8, 2210 (2017).

18. S. Korganbayev, T. Ayupova, M. Sypabekova, A. Bekmurzayeva, M. Shaimerdenova, K. Dukenbayev, C. Molardi, and D. Tosi, Opt. Express 26, 18708 (2018). 